\title{
The mechanical performance of sugar palm fibres (Ijuk) reinforced phenolic composites
}

\begin{abstract}
Sugar palm fibres are one of the natural fibres which have many features and need further study to understand their properties. The aim of this work is to investigate the flexural, compressive and impact properties of sugar palm fibres reinforced phenolic composites. Sugar palm fibres were used as a filler (particle size $150 \mu \mathrm{m}$ ) and with loading of $0,10,20$, 30 , and $40 \mathrm{vol} . \%$. The fibres were treated by sea water and then fabricated into composites by hot press technique. Flexural, compressive, and impact tests were carried out as per ASTM D790, ASTM D695-08a, and ASTM D256 standards, respectively. Scanning electron microscopy (SEM) was used to investigate the morphology and the interfacial bonding of the fibres-matrix in composites. The results show that the mechanical properties of the composites improve with the incorporation of fibres. The composite of $30 \mathrm{vol} \%$ particle loading exhibit optimum values which are $32.23 \mathrm{MPa}, 61.66 \mathrm{MPa}$, and $4.12 \mathrm{~kJ} / \mathrm{m} 2$ for flexural, compressive, and impact strength, respectively. This was because good compatibility of fibre-matrix bonding. Consequently, sugar palm fibre is one of the prospective fibres and could be used as a potential resource to reinforcement polymer composite.
\end{abstract}

Keyword: Composites; Compressive; Flexural; Impact; Phenolic resin; Sugar palm fibres 